\title{
Investigating Molecule-plasmon Interactions in Chemically-functionalized Metal Nanoparticles Using Monochromated EELS
}

\author{
P. Abellan ${ }^{1}$, P.Z. El-Khoury ${ }^{2}$, F.S. Hage, ${ }^{1}$ J. Cottom, ${ }^{3}$ A.G. Joly,${ }^{2}$ W.P. Hess, ${ }^{2}$ R. Brydson ${ }^{3}$ and Q.M. \\ Ramasse $^{1}$ \\ 1. SuperSTEM Laboratory, SciTech Daresbury Campus, Daresbury, U.K. \\ 2. Physical Sciences Division, Pacific Northwest National Laboratory, Richland, U.S.A. \\ 3. School of Chemical and Process Engineering, University of Leeds, Leeds, U.K.
}

Metal nanostructures can be used to detect, image, and identify molecules with high sensitivity and selectivity using tools of (non-)linear optical spectroscopy [1]. Slight nanometric variations in the morphology and distribution of metal particles can completely alter the plasmonic response of the local electric field interacting with nearby molecules. Indeed, the interplay and coupling of molecules to plasmons is known to be complex and the exact mechanisms behind a plethora of phenomena of mixed molecular-metallic/plasmonic origin are not well understood. In this regard, the commonly adopted practice of correlating structural/topographic images of plasmonic nanostructures with near-field optical spectra is often insufficient. Among other difficulties, the intense scattering arising from the metal constitutes a challenging technical issue for the study of molecule to metal or metal to molecule charge transfer (CT) states by optical methods. Notably, the direct measurement of the modified optical absorption of molecules on plasmonic nanoparticles has only been reported within the last year [2]. Novel approaches that can be used to gain access to the molecular electronic and vibrational states and to the plasmonic response of plasmonic particles on a nanometer length scale could yield a paradigm shift in thinking about molecule-plamon interactions and surface-enhanced optical spectroscopies.

Using electrons in a Cs-corrected scanning transmission electron microscope (STEM), unrivalled spatial resolution for measuring the plasmonic response of nanoparticles is achieved. Furthermore, electron energy loss spectroscopy (EELS) has already provided spectral evidence of CT states and classical/quantum plasmonic resonances in dimers of cubic metal particles containing an organic dielectric junction.[3] In this work, we demonstrate the use of monochromated EELS in a Nion UltraSTEM 100MC Hermes microscope [4], with practical energy resolution of $12 \mathrm{meV}$, and operated at $60 \mathrm{kV}$ to simultaneously visualize the local electric fields associated with plasmonic excitation and to probe aromatic thiols in chemically-functionalized metal particles. We will present our later results aimed at finding experimental conditions for identifying molecular reporters (eg. 4,4'dimercaptostilbene, $\mathrm{C}_{14} \mathrm{H}_{12} \mathrm{~S}_{2}$, DMS - see Fig. 1A) and discuss the effect of different microscope settings and possible TEM sample preparation techniques. We will also explore the interaction between molecules and plasmons on the nanoscale and discuss some of the limitations we have found for such simultaneous spectral recording and mapping of vibrational and plasmonic modes (see Fig. 1B and 1C). We support some of our observations with numerical simulations using finite-element method implemented in commercial software (COMSOL Multiphysics v5.2). We will discuss plausible identification of different resonances and their spatial variances as well as experimental and theoretical limitations of our evolving approach to nanoscale combined chemical and electric field imaging [5]. 


\section{References:}

[1] Pettinger et al, N. Annu. Rev. Phys. Chem. 63 (2012), p. 379; Le Ru \& Etchegoin, Annu. Rev. Phys. Chem. 65 (2012), p. 65.

[2] Darby et al, Nat. Photonics 10 (2016), p. 40.

[3] Tan et al, Science 343 (2014), p. 1496.

[4] Krivanek et al, Nature Letter 514 (2014), p. 209; R. Brydson, Nature 514 (2014), p. 177.

[5] SuperSTEM is the UK EPSRC National Facility for Aberration-Corrected STEM, supported by the Engineering and Physical Science Research Council. PZE acknowledges support from the Laboratory Directed Research and Development Program at Pacific Northwest National Laboratory (PNNL). WPH and AGJ are supported by the US Department of Energy (DOE), Office of Science, Office of Basic Energy Sciences, Division of Chemical Sciences, Geosciences \& Biosciences.
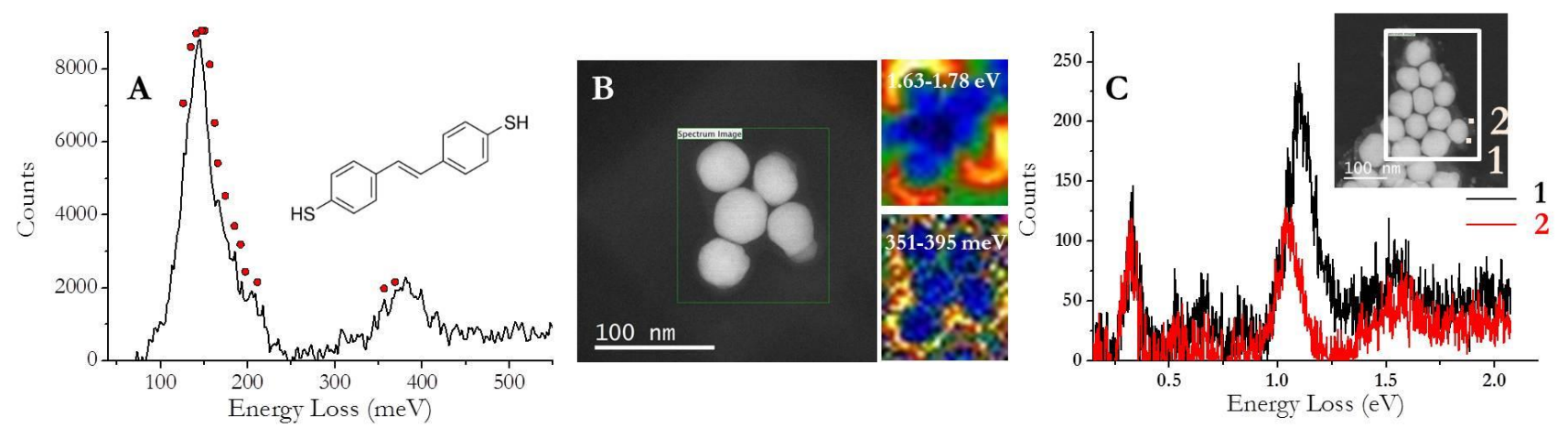

Figure 1. A) Vibrational modes of DMS, with vibrations (IR and Raman active) indicated by red circles. The most intense peak is assigned to the C-S stretching (134meV) and aromatic C-H in-plane rocking $(147 \mathrm{meV})$ vibrations. B) Simultaneous mapping of aromatic $\mathrm{C}-\mathrm{H}(360 \mathrm{meV})$ from surface bonded molecules and Ag nanoparticle surface plasmon modes. C) Vibrational response and plasmonic resonances measured in aloof geometry from locations 1 and 2 (see inset). The energy resolution was 12 $\mathrm{meV}$ (full-width at half-maximum of the zero-loss peak through the support). 